\title{
Vortex Dynamics in Ferromagnetic Nanoelements Observed by Micro-Hall Probes
}

\author{
T. ŠČepka*, J. Šoltýs, M. Precner, J. Fedor, J. Tóbik, D. Gregušová, F. Gucmann, \\ R. KÚdela, V. CAMBel
}

Institute of Electrical Engineering, Slovak Academy of Sciences, 84104 Bratislava, Slovakia

\begin{abstract}
In this work we measure the nucleation and annihilation of magnetic vortices in Pacman-like (PL) micromagnets prepared from Permalloy $\left(\mathrm{Ni}_{81} \mathrm{Fe}_{19}, \mathrm{Py}\right)$ at $77 \mathrm{~K}$. Lateral dimensions of explored objects are $\leq 1 \mu \mathrm{m}$ with thickness of about $40 \mathrm{~nm}$. The micromagnets are located directly on the high-sensitive micro-Hall probe based on GaAs/AlGaAs heterostructure by lift-off process. Experiments show good agreement of the magnetization reversal with the micromagnetic simulation. Other shapes of micromagnets are also considered to obtain more precise picture of the vortex dynamics.
\end{abstract}

DOI: 10.12693/APhysPolA.126.390

PACS: 81.16.Nd, 75.60.Jk, 74.25.Ha

\section{Introduction}

Current developments in controlled manipulation of magnetic domains in ferromagnetic structures open opportunities for unique memory architectures with higher storage density, faster performance, and lower power consumption to write and read the information $[1,2]$. The benefit of the magnetic structures with specific ordering is their ability to store two bits of information simultaneously (in terms of chirality and polarity of the magnetic vortex).

Hall magnetometry based on micro-Hall probes is a powerful technique used for acquiring quantitative information on local stray field of individual ferromagnetic objects, which includes observations of vortex dynamics. From the experiments, it was clear that the magnetization reversal of disks includes vortex nucleation, propagation, and annihilation [3-5]. However, the detailed mechanism of vortex formation remains still an open problem.

In our previous work based on micromagnetic calculations, Pacman-like (PL) nanomagnet has been designed and its magnetic properties have been calculated $[6,7]$. It is shown that ground states can be controlled only by applying in-plane fields and the relation between the element with broken rotational symmetry and the direction of applied field has to be considered.

\section{Experiment}

Hall crosses that serve as high-sensitive probes were fabricated from a GaAs/AlGaAs heterostructure. At first Hall crosses and contact leads were defined by optical lithography followed by wet chemical etching $\left(1 \mathrm{H}_{3} \mathrm{PO}_{4}: 2 \mathrm{H}_{2} \mathrm{O}_{2}: 8 \mathrm{H}_{2} \mathrm{O}\right.$ system $)$. In the second step a

\footnotetext{
*corresponding author; e-mail: tomas.scepka@savba.sk
}

miniaturization of the fabricated crosses was performed by EBL lithography using a PMMA resist that was exposed by the dose of $250 \mu \mathrm{C} / \mathrm{cm}^{2}$ at $20 \mathrm{kV}$ and wet chemical etching by the same system. In the subsequent steps, PL micromagnet was patterned by EBL lithography (dose of $110 \mu \mathrm{C} / \mathrm{cm}^{2}$ at $20 \mathrm{kV}$ ), e-beam evaporation of $40 \mathrm{~nm}$ Py, and freed by standard lift-off process.

Only one PL was patterned per cross to enable singleelement observation. The hysteresis loops were measured by applying an in-plane magnetic field and recording the resulting Hall voltage while biasing the device with a dc current of $10 \mu \mathrm{A}$. Measurements were carried out at room and $77 \mathrm{~K}$ temperatures, however due to noise only lowertemperature characteristics are presented.

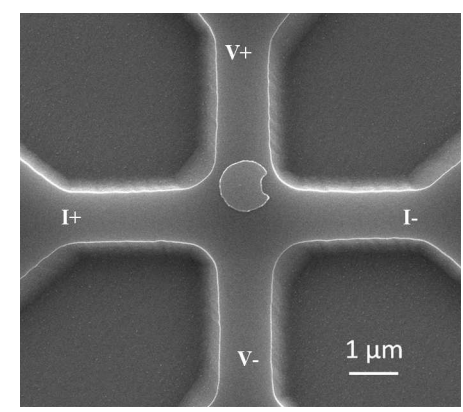

Fig. 1. Scanning electron microscope micrograph of miniaturized Hall probe with Pacman-like micromagnet.

Figure 1 shows fabricated micro-Hall probe with the PL micromagnet located on the probe. Diameter of the PL element is $1 \mu \mathrm{m}$ and an active region of the probe is approximately $1 \times 1 \mu \mathrm{m}^{2}$. The micromagnet is shifted from the central position to improve the measured signal. Best resolution is achieved for Hall configuration when current flows between leads $I+$ and $I-$, and voltage drop is measured between leads $V+$ and $V-$. 


\section{Results and discussion}

Experimental magnetization reversal trace for the $\mathrm{PL}$ micromagnet (Fig. 1) is shown in Fig. 2a. Jumps in the stray field correspond to significant changes of the magnetic state (vortex nucleation and annihilation, etc.). Such changes are directly connected with the abrupt redistribution and/or change in the exchange and dipole energies of the system. Smooth change of the signal can be attributed to the smooth shift of the magnetization configuration (shift of the vortex, c-state, s-state, etc.) within the ferromagnet.
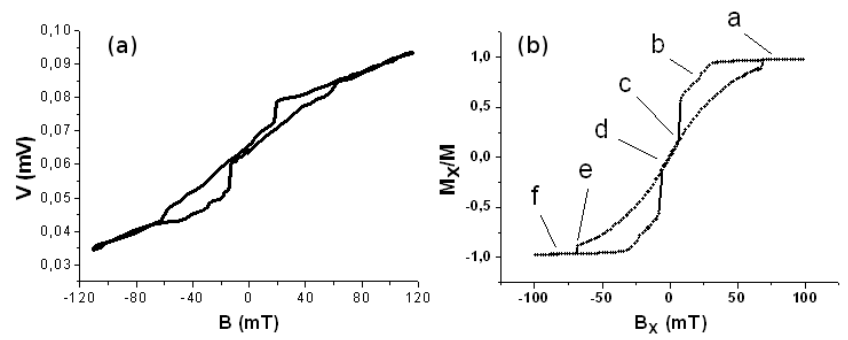

Fig. 2. a) Magnetization reversal of PL micromagnet measured in the Hall configuration at $77 \mathrm{~K}$. The in-plane magnetic field applied at the angle of $15^{\circ}$ with respect to PL symmetry axis, b) simulated magnetization reversal of the PL element.

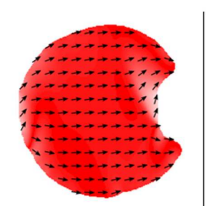

(a) $80 \mathrm{mT}$

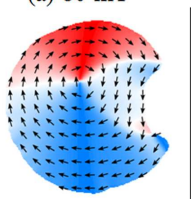

(d) $-12 \mathrm{mT}$

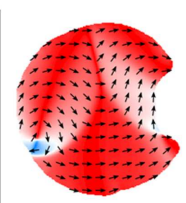

(b) $21 \mathrm{mT}$

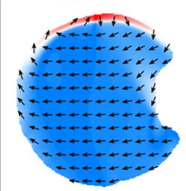

(e) $-67 \mathrm{mT}$

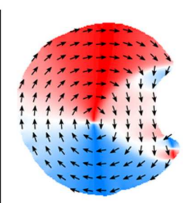

(c) $6 \mathrm{mT}$

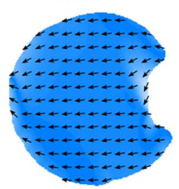

(f) $-80 \mathrm{mT}$
Fig. 3. Images of local magnetization obtained by micromagnetic simulation.

Hysteresis loop in Fig. 2a contains two significant jumps for both external field directions. To evaluate the voltage drop of the jumps, the measurement for a particular angle was repeated many times. Two dominant values of the voltage drop were observed and we suppose that they correspond to two values of the PL-micromagnet chirality.

Figure 2b shows simulated magnetization reversal using OOMMF software package [8] (zero-temperature simulation). The simulation was performed on $1-\mu \mathrm{m}$ element that contains similar boundary defects as the fabricated PL micromagnet. Magnetic field in the simulation was applied at the angle of $7.5^{\circ}$ with respect to $x$-axis, which is close to the angle $15^{\circ}$ selected in the experiment. Important points in the simulated loop are labelled by let- ters $(\mathrm{a}-\mathrm{f})$, for which corresponding magnetization images are shown in Fig. 3. The sequence illustrates reordering of the magnetic pattern. At $21 \mathrm{mT}$ vortex nucleates, the biggest jump at $6 \mathrm{mT}$ corresponds to the jump of the vortex to the central position. For the fields between $6 \mathrm{mT}$ and $-67 \mathrm{mT}$ the vortex propagates perpendicular to the applied magnetic field. The annihilation of the vortex appears at $-67 \mathrm{mT}$.

\section{Conclusions}

In this work, we have explored magnetic properties of 1- $\mu \mathrm{m}$ Pacman-like ferromagnet in external magnetic field using micro-Hall probe. In the experiment we have focused on the nucleation field of the vortex and we have found that there are two levels of the Hall-voltage drop that depend on vortex chirality. The shape of the ferromagnet in the micromagnetic simulation was identical to the shape of the fabricated one. Therefore, the simulation can explain qualitatively the experimental magnetization loop. However, due to the temperature differences between the experiment and the simulation there is a slight discrepancy in the hysteresis loops. We suppose that for lower PL dimensions even more precise picture of the vortex dynamics should be seen within experiment presented.

\section{Acknowledgments}

This work was supported by Slovak Grant Agency APVV, projects APVV-0088-12 and VVCE-0058-07, by VEGA project $2 / 0037 / 12$, and by the Research \&Development Operational Program funded by the ERDF, "CENTE", ITMS code 26240120011(0.5).

\section{References}

[1] S.S.P. Parkin, M. Hayashi, L. Thomas, Science 320, 190 (2008).

[2] C.A. Ross, H.I. Smith, T. Savas, M. Schattenburg, M. Farhoud, M. Hwang, M. Walsh, M.C. Abram, R.J. Ram, J. Vac. Sci. Technol. B 17, 3168 (1999).

[3] T.M. Hengstmann, D. Grundler, Ch. Hezn, D. Heintmann, J. Appl. Phys. 90, 6542 (2001).

[4] M. Rahm, M. Schneider, J. Biberger, R. Pulwey, J. Zweck, D. Weiss, V. Umansky, Appl. Phys. Lett. 82, 4110 (2003).

[5] G. Mihajlovic, M.S. Patrick, J.E. Pearson, V. Novosad, S.D. Bader, M. Field, G.J. Sullivan, A. Hoffmann, Appl. Phys. Lett. 96, 112501 (2010).

[6] V. Cambel, G. Karapetrov, Phys. Rev. B 84, 014424 (2011).

[7] J. Tobik, V. Cambel, G. Karapetrov, Phys. Rev. B 86, 134433 (2012).

[8] M.J. Donahue, D.G. Porter, OOMMF User's Guide, Version 1.0 , Technical Report No. NISTIR 6376, National Institute of Standards and Technology, Gaithersburg, MD (1999). 